\title{
(C) OPEN ACCESS \\ Variations in specialist palliative care referrals: findings from a population-based patient cohort of acute myeloid leukaemia, diffuse large B-cell lymphoma and myeloma
}

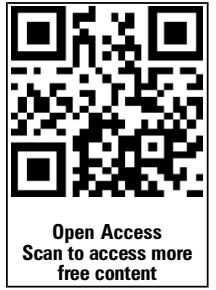

\author{
D A Howell, ${ }^{1} \mathrm{H}-\mathrm{I}$ Wang, ${ }^{1}$ E Roman, ${ }^{1}$ A G Smith, ${ }^{1}$ R Patmore, ${ }^{2}$ \\ M J Johnson, ${ }^{3}$ A C Garry, ${ }^{4}$ M R Howard ${ }^{4}$
}

${ }^{1}$ Epidemiology and Cancer Statistics Group, Department of Health Sciences, University of York, York, North Yorkshire, UK ${ }^{2}$ Queens Centre for Oncology, Castle Hill Hospital, Hull, East Yorkshire, UK

${ }^{3}$ Hull York Medical School, The University of Hull, Hull, East Yorkshire, UK

${ }^{4}$ York Teaching Hospital NHS Foundation Trust, York, North Yorkshire, UK

\section{Correspondence to} Dr D A Howell, Epidemiology and Cancer Statistics Group, Department of Health Sciences, University of York, York, North Yorkshire Y010 5DD, UK debra.howell@ecsg.york.ac.uk

Received 8 August 2013 Revised 19 December 2013 Accepted 19 January 2014 Published Online First

19 February 2014

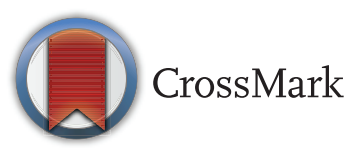

To cite: Howell DA, Wang $\mathrm{H}$ I, Roman E, et al. BMJ Supportive \& Palliative Care 2015:5:496-502.

\section{ABSTRACT}

Objective To develop and implement a methodology for capturing complete haematological malignancy pathway data and use it to identify variations in specialist palliative care (SPC) referrals.

Methods In our established UK populationbased patient cohort, 323 patients were diagnosed with acute myeloid leukaemia, diffuse large B-cell lymphoma or myeloma between May 2005 and April 2008, and died before April 2010. A day-by-day calendar approach was devised to collect pathway data, including SPC referrals, to supplement routinely collected information on clinical presentation, diagnosis, treatment, response, and date and place of death.

Results 155 (47.9\%) of the 323 patients had at least one SPC referral. The likelihood of referral increased with survival (OR 6.58, 95\% Cls 3.32 to 13.03 for patients surviving $\geq 1$ year compared to $\leq 1$ month from diagnosis), and varied with diagnosis (OR 1.96, Cls 1.15 to 3.35 for myeloma compared to acute myeloid leukaemia).

Compared to patients dying in hospital, those who died at home or in a hospice were also more likely to have had an SPC referral (OR 3.07, Cls 1.59 to 5.93 and $4.74, \mathrm{Cls} 1.51$ to 14.81 , respectively). No associations were found for age and sex.

Conclusions Our novel approach efficiently captured pathway data and SPC referrals, revealing evidence of greater integration between haematology and SPC services than previously reported. The likelihood of referral was much higher among those dying outside hospital, and variations in practice were observed by diagnosis, emphasising the importance of examining diseases individually.

\section{INTRODUCTION}

Patients with haematological malignancies are reported to be less likely to receive input from specialist palliative care (SPC) or hospice services than those with other cancers. ${ }^{1}{ }^{2}$ These diseases are associated with uncertainty about when SPC referrals should be made ${ }^{3}$; and they have significantly shorter intervals between referral and death compared to other cancers. ${ }^{4} 5$ Patients with haematological malignancies are also more likely to die in hospital ${ }^{6-8}$; a finding that is consistent across the disease spectrum despite the very diverse individual diagnostic subtypes. ${ }^{9}$ Although an evidence base is beginning to emerge about these differences, explanations are still largely based on speculation and anecdote. In the UK, the Department of Health acknowledges the challenges associated with the delivery of end-of-life and palliative care in patients with haematological cancers, and in 2003, published guidance recommending further integration between clinical haematology and SPC services. ${ }^{10}$

Haematological malignancies are common, representing 1 in 10 of all cancers in the developed world. ${ }^{11} 12$ They are complex diseases, ranging from those that are acute in onset and often associated with poor survival, such as acute myeloid leukaemia (AML); to those that often follow a pathway of care closer to chronic disease than malignancy, such as myeloma. Treatments vary markedly and may include a 'watch and wait' approach to care (where the patient is 
actively monitored and only treated at the time of disease progression, or when they become particularly symptomatic), intensive or non-intensive chemotherapy, radiotherapy, bone marrow transplantation or antibody therapy. Many patients also receive supportive care (particularly blood products), which may be delivered alone or in combination with other treatments. Surgery is rarely a curative option in these diseases, although it may be used, for example, to stabilise fractures caused by bone disease in patients with myeloma. Finally, multiple lines of chemotherapy treatment may be given, particularly for the diseases that follow a continuous remitting/relapsing course.

Treatment may be given with curative intent, lifeprolonging intent (for diseases, such as myeloma which is generally considered incurable at diagnosis), or for disease and symptom control. In this respect, the definition of 'palliative care' and the 'transition' from an active, life prolonging to a palliative approach to care - which is recognised as being complicated generally ${ }^{13}{ }^{14}$-is particularly complex in these diseases. Additionally, the symptom burden associated with haematological malignancies is considered comparable to that of other cancers, with physical symptoms including fatigue, fever, bleeding, drowsiness, oral stomatitis and pain; and psychological symptoms including adjustment disorder, mood disorder and anxiety. ${ }^{15-20}$ These problems arise due to both the disease processes and associated complications, as well as the side-effects and toxicities of treatments. ${ }^{21}$ No correlation has been reported between the presence of symptoms and the likelihood of access to SPC services as a consequence of these. ${ }^{19} 22$

There is little existing evidence about the factors determining differences in end-of-life care in patients with haematological cancers. This potentially inhibits identification of changes that could be introduced to ensure that patients receive the most appropriate and timely care, in a place of their choosing. Built on the pre-existing infrastructure of an established population-based patient cohort, the current research programme was instigated to address these issues. Our overarching aim was to develop and implement a methodology to capture detailed information about the care pathways (providers/settings) and transitions (curative/life-prolonging and palliative) of patients with specific haematological malignancies, during the period of time between diagnosis and death. The current paper describes the methodology, presents an overview of the dataset, and examines variations in SPC referral by age, sex, diagnostic sub-type, time from diagnosis to death, and place of death.

\section{METHODS}

This programme of work was conducted within the existing framework of the Haematological Malignancy Research Network (http://www.HMRN.org), an on-going patient cohort covering a population of 3.6 million (>2000 newly diagnosed haematological malignancies each year) that is broadly representative of the UK as a whole. ${ }^{23}{ }^{24}$ Established in 2004, HMRN is a collaboration between a clinical haematology network, researchers at the University of York and the Haematological Malignancy Diagnostic Service (http://www.HMDS.info), which diagnoses all haematological malignancies in the area coding to the latest WHO classification scheme (currently ICD-O-3). ${ }^{25}$ HMRN operates with Section 251 support under the National Health Service (NHS) Act 2006, enabling the Health and Social Care Information Centre (HSCIC) to provide us with nationwide information on deaths, subsequent cancer registrations, and Hospital Episode Statistics (HES). Additionally, all patients have full treatment, response and outcome data abstracted from their medical records by trained nurses operating to clinical trial standards.

Within HMRN, patients with haematological malignancies are treated at 14 hospitals. For the current study, we identified all patients newly diagnosed with AML, diffuse large B-cell lymphoma (DLBCL) and myeloma between 1 May 2005 and 30 April 2008, who received their first treatment at one of two large teaching hospitals, and were deceased by 30 April 2010 (reflecting a minimum of 2 years and maximum of 5 years follow-up). Patients living in the catchment area of these two hospitals may access SPC services in the hospital (in-patient and out-patient) and/or the community setting; and hospice care is also available.

For this project, HMRN's routinely collected data were supplemented by more detailed information abstracted from hospital records and community SPC records, using a day-by-day calendar approach spanning the entire time from diagnosis to death. This included details of all specific hospital events (outpatient appointments, in-patient episodes, medical specialities managing care and clinical decisions); SPC contacts (referrals to the hospital and/or community teams, type and frequency of contact and input); and details of death (preferred and actual place of care and death). Figure 1 summarises the data collection framework, with blue boxes indicating the routine data collection which takes place for all HMRN patients and green boxes showing the additional pathway data collected for patients in this study.

Data were entered into a calendar and event-based relational database, with each key event having the capacity to store information on multiple sub-events. This permitted information, such as in-patient episodes, to be recorded alongside the specialists providing care and other important sub-events including, for example, clinical decisions (such as transitions in care or resuscitation status). This enabled detailed patientspecific pathways to be constructed and manipulated to highlight specific events and activities of interest. Analyses were conducted with SAS (V.9.3), ${ }^{26}$ and logistic regression was used to compare differences 


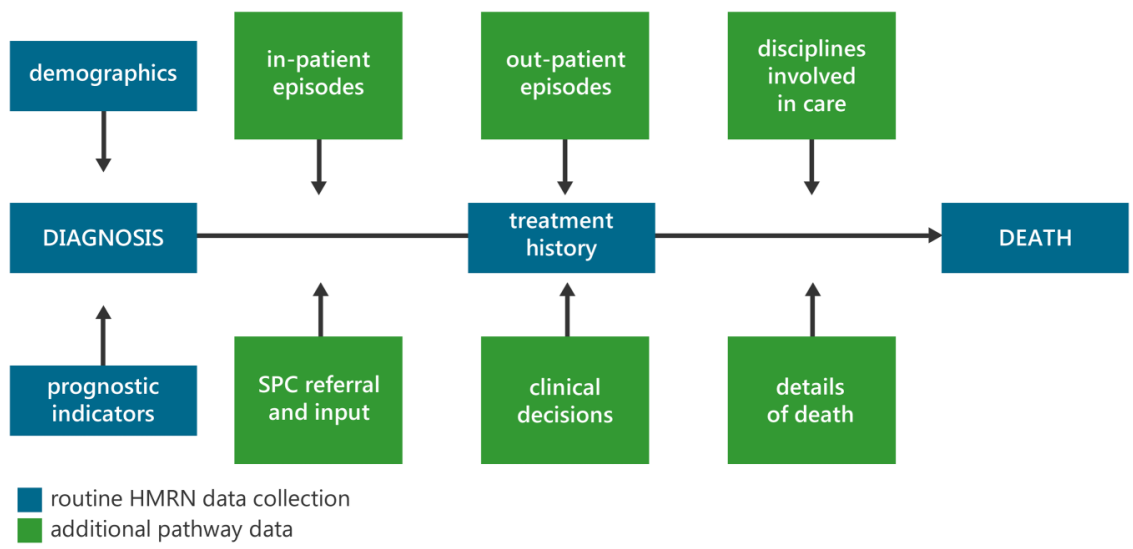

Figure 1 Summary of data.

between patients who received SPC and those who did not.

\section{RESULTS}

Of the 323 patients who were diagnosed and died during the study period, 107 (33.1\%) had AML, 102 $(31.6 \%)$ DLBCL and 114 (35.3\%) had myeloma (table 1). Overall, 155 (47.9\%) had at least one SPC referral, and there were no differences between those that were referred and those that were not in terms of age or sex. Significant differences in referral patterns were evident, however, by disease sub-type; patients with myeloma being almost twice as likely to have an SPC referral (OR 1.96, CIs 1.15 to 3.35 ) as those with AML. The likelihood of referral also increased with longer survival; patients surviving 1 year and above, for example, were over six times more likely to be referred (OR 6.58, 95\% CIs 3.32 to 13.03) than those dying within a month of diagnosis. Being referred to the SPC team was also significantly associated with place of death, and those dying at home were three times more likely to be referred to SPC services (OR 3.07, CIs 1.59 to 5.93) and those dying in a hospice almost five times more likely (OR 4.74, CIs 1.51 to 14.81 ) compared with those dying in hospital. Figure 1 Summary of data

With respect to the type of SPC input, referral to the community team was slightly more common than

Table 1 Patient characteristics by SPC referral

\begin{tabular}{|c|c|c|c|c|}
\hline & $\begin{array}{l}\text { Total } \\
\text { n (\%) }\end{array}$ & $\begin{array}{l}\text { No SPC referral } \\
\mathrm{n}(\%)\end{array}$ & $\begin{array}{l}\text { SPC referral } \\
\text { n (\%) }\end{array}$ & OR (95\% CI) \\
\hline Total & $323(100)$ & $168(100)$ & $155(100)$ & \\
\hline \multicolumn{5}{|l|}{ Sex } \\
\hline Male & $178(55.1)$ & $92(54.8)$ & $86(55.5)$ & 1 \\
\hline Female & $145(44.9)$ & $76(45.2)$ & $69(44.5)$ & $0.97(0.63$ to 1.51$)$ \\
\hline \multicolumn{5}{|l|}{ Age, years } \\
\hline$\leq 60$ & $58(18.0)$ & $28(16.7)$ & $30(19.3)$ & 1 \\
\hline $61-74$ & $122(37.8)$ & $61(36.3)$ & $61(39.4)$ & $0.93(0.50$ to 1.74$)$ \\
\hline $75+$ & $143(44.2)$ & $79(47.0)$ & $64(41.3)$ & $0.76(0.41$ to 1.34$)$ \\
\hline \multicolumn{5}{|l|}{ Diagnosis } \\
\hline AML & $107(33.1)$ & $61(36.3)$ & $46(29.7)$ & 1 \\
\hline DLBCL & $102(31.6)$ & $61(36.3)$ & $41(26.4)$ & $0.89(0.51$ to 1.55$)$ \\
\hline Myeloma & $114(35.3)$ & $46(27.4)$ & $68(43.9)$ & 1.96 (1.15 to 3.35$)$ \\
\hline \multicolumn{5}{|c|}{ Time from diagnosis to death (months) } \\
\hline$\leq 1$ & $75(23.2)$ & $59(35.1)$ & $16(10.3)$ & 1 \\
\hline $1-6$ & $83(25.7)$ & $47(28.0)$ & $36(23.2)$ & $2.82(1.40$ to 5.70$)$ \\
\hline $6-12$ & $62(19.2)$ & $25(14.9)$ & $37(23.9)$ & $5.46(2.58$ to 11.56$)$ \\
\hline $12+$ & $103(31.9)$ & $37(22.0)$ & $66(42.6)$ & $6.58(3.32$ to 13.03$)$ \\
\hline \multicolumn{5}{|l|}{ Place of death } \\
\hline Hospital & $240(74.3)$ & $138(82.1)$ & $102(65.8)$ & 1 \\
\hline Home & $49(15.2)$ & $15(8.9)$ & $34(21.9)$ & 3.07 (1.59 to 5.93$)$ \\
\hline Hospice & $18(5.6)$ & $4(2.4)$ & $14(9.0)$ & $4.74(1.51$ to 14.81$)$ \\
\hline Nursing home & $16(4.9)$ & $11(6.6)$ & $5(3.2)$ & $0.62(0.21$ to 1.83$)$ \\
\hline
\end{tabular}

$\mathrm{AML}$, acute myeloid leukaemia; DLBCL, diffuse large B-cell lymphoma; SPC, specialist palliative care. 
Type of SPC referral

\begin{tabular}{llllll} 
& $\begin{array}{l}\text { Total } \\
\mathrm{n}(\%)\end{array}$ & SPC referral $\mathrm{n}(\%)$ & $\begin{array}{l}\text { Hospital } \\
\mathrm{n}(\%)\end{array}$ & $\begin{array}{l}\text { Community } \\
\mathrm{n}(\%)\end{array}$ & $\begin{array}{l}\text { Hospital and } \\
\text { community }(\mathrm{n} \%)\end{array}$ \\
\hline Total & $323(100)$ & $155(47.9)$ & $100(31.0)$ & $117(36.2)$ & $62(19.2)$ \\
AML & $107(100)$ & $46(43.0)$ & $21(19.6)$ & $40(37.4)$ & $15(14.0)$ \\
DLBCL & $102(100)$ & $41(40.2)$ & $26(25.5)$ & $30(29.4)$ & $15(14.7)$ \\
Myeloma & $114(100)$ & $68(59.6)$ & $53(46.5)$ & $47(41.2)$ & $32(28.1)$ \\
\hline
\end{tabular}

AML, acute myeloid leukaemia; DLBCL, diffuse large B-cell lymphoma; SPC, specialist palliative care.

referral to the hospital team, although we found that $62(19.2 \%)$ patients received support from both hospital and community services (table 2). Different referral practices were observed by diagnostic sub-type; patients with AML being more likely to be referred to the community than the hospital SPC team $(37.4 \%$ and $19.6 \%$, respectively). Myeloma patients were somewhat more likely to be referred to hospital than community services (46.5\% compared with $41.2 \%$, respectively), although a large proportion of these patients $(28.1 \%)$ were referred to both services.

\section{DISCUSSION}

This is the first study to examine referral to SPC services in patients with AML, DLBCL and myeloma. Importantly, we found evidence of greater integration between clinical haematology teams and SPC services than expected, with almost half the patients having at least one referral. Variations with diagnosis were, however, observed, and patients with myeloma were more likely to be referred than those with AML or DLBCL. We found important variations in referral practices by duration of time from diagnosis to death, with the likelihood of referral increasing with longer survival. SPC referral was clearly associated with place of death, and those referred were less likely to die in hospital. Evidence of different types of SPC referral (to the hospital or community team) was also noted by diagnostic sub-type.

\section{Strengths/weaknesses of study}

With more than 300 patients, our study is the largest in the UK and one of the largest in the world to examine SPC referrals specifically in patients with haematological cancers. Covering a population of 3.6 million, our patient cohort (HMRN) is generalisable to the UK, and provides a robust, well-established infrastructure for research. ${ }^{23} 24 \mathrm{~A}$ major strength of HMRN is that all diagnoses are routinely coded to ICD-O- $3,{ }^{25}$ meaning that we were able to examine variations between specific sub-types of haematological cancers, which is of particular importance given that these diseases can differ so markedly, with some being particularly indolent and others aggressive. This characteristic has an impact on the duration of survival, which is recognised as being associated with likelihood of SPC referral; longer survival equating to increased chance of referral. ${ }^{27-30}$ The differences identified between the diseases included in this study demonstrate the importance of being able to examine specific sub-types in this way.

A further strength of our study is the enormous breadth and depth of data that were collected for each individual patient in the cohort. The complete treatment pathway from diagnosis to death was examined, therefore, encompassing hospital and community SPC input during this time. Data routinely collected on all HMRN patients to clinical trial standards formed the basis from which the treatment pathways were developed. These were supplemented by specific eventbased information collected from detailed searching of numerous sources, including paper and computerised medical records. As a result of this multifaceted approach, we are extremely confident that the vast majority of SPC referrals were identified.

Although our on-going study may have raised awareness of the importance of integration between clinical haematology and SPC services in the study area, much of the data collection occurred retrospectively, and it is unlikely that this will have unduly influenced referral practices. The study was conducted in two large teaching hospitals, and while it is probable that there are some differences in practice in other geographical locations, we expect that our results are likely to be generally illustrative of other areas. One weakness of the study is that the maximum duration of the follow-up period was only 5 years. This means that the SPC contacts for myeloma and relapsed DLBCL may have been underestimated, although it is unlikely to have biased the results for AML, which is associated with particularly poor survival. Finally, as our study focused on specific haematological malignancies, we do not have comparators for other cancers (or indeed other haematological cancers), so cannot comment on relative differences between disease types.

\section{Comparison with other studies}

A number of studies examining SPC referrals have included patients with haematological malignancies. ${ }^{1} 22 \quad 27-36$ We observed much greater integration between clinical haematology and SPC services than 
was identified in the majority of these studies in the UK or elsewhere, and whether examining hospital or community referrals. The exception to this was a large Australian study. ${ }^{28}$ It is difficult, however, to directly compare our findings with the results from existing studies, initially because of the use of widely varying methods of data collection, including interviews and questionnaires, as well as examination of medical records and database linkages, for example, to healthcare billing data. These studies have also examined involvement of SPC services in different settings (hospital or community), or with respect to specific services (such as Hospice at Home or Palliative Home Care), and some have also included only very small numbers of patients with haematological malignancies. Previously reported studies have not been able to categorise diseases as precisely as we have done and, instead, have defined 'haematological diseases' as a single group (including all leukaemias, lymphomas and myeloma), or have examined all types of lymphoma together and/or all types of leukaemia. More rarely, some specificity has been imposed by focussing on particular disease types, such as acute leukaemias. Finally, the situation is further complicated by the varied service provision inherent within and between countries, as well as different cultural practices.

Our results concur with other studies with respect to the association between longer survival and increasing likelihood of SPC referral, ${ }^{27-30}$ and also the association between SPC referral and fewer hospital deaths for haematology patients and cancer patients in general. ${ }^{28} 303537$

\section{Meaning of study: explanations and implications for clinicians and policy makers}

The differences we observed by diagnostic subgroup are comprehensible with respect to individual disease characteristics. Myeloma patients were referred more frequently to SPC services than patients with AML and DLBCL. By contrast with some of the other haematological malignancies, myeloma has many potential complications at diagnosis and relapse, including bone disease and fractures, severe and complex pain and renal failure. These problems often require support from a multidisciplinary team of clinicians with expertise in these areas. SPC practitioners are widely recognised and acknowledged for their skills in the management of complex issues such as these, particularly within the context of dealing with a new or relapsed cancer diagnosis, and many of the SPC referrals we identified were undoubtedly made in order to capitalise on these skills. ${ }^{38} 39$

The high proportion of SPC referrals to community services in patients with AML may have occurred due to the lack of treatment options (beyond non-intensive chemotherapy and blood transfusions) available to older people with these diseases. This may mean that the terminal nature of the disease is recognised earlier in the care pathway (indeed at diagnosis), allowing community services to be put in place well in advance of the patient's deterioration. Further referrals across diseases are likely to have been made for a number of reasons, including: management of complex symptoms, such as the severe toxicity resulting from intensive treatment of AML in younger patients, or peripheral neuropathy, which is a common side effect of the chemotherapy used to treat myeloma and also lymphoma; provision of terminal care; and discussions about preferred place of care and death.

As mentioned above, SPC referrals may occur more often in patients who survive longer because there is adequate time to recognise that curative/lifeprolonging treatment options are diminishing, and there is less chance of prolonged survival. The transition in this context is likely to occur over a period of time, rather than being associated with sudden deterioration and death. Furthermore, we noted fluctuations in SPC involvement over time, with services withdrawing as particular issues resolve, and then re-engaging later if new concerns arise. These multiple and often overlapping episodes also provided evidence of interservice referrals and activities between hospital and community teams.

SPC referral is undoubtedly associated with a reduction in hospital deaths, and this has clear implications for practitioners, who are often the gatekeepers of these services, as well as policy makers who are able to encourage referrals at a national level. What is unclear, however, is whether SPC referrals instigate discussions about preferred place of care and death, and the possibility of home/hospice death, or whether the patient has decided that their preferred place of care and death is home/hospice, and the referral was made to the service to facilitate this. Nonetheless, home deaths are clearly more likely to occur in patients with SPC referrals, and so, this information is important when considering how best to achieve preferred place of death when a patient and their family decides that this is not hospital.

\section{Unanswered questions and future research}

Haematological malignancies are complex, and our population-based patient cohort is now generating evidence which is facilitating a better understanding of palliative and end-of-life care in these diseases. We observed a high rate of SPC referrals, but this does not mean that patients who were not referred had unmet needs; it is possible that these patients did not have particularly complex problems that required involvement from SPC services, and so, were managed solely by the clinical haematology team in the hospital or by general practitioners and district nurses in the primary care setting. Conducting similar studies in different geographical areas would be useful to further assess variations in practice. Comparing our results with a cohort 
examining non-haematological cancers would also promote understanding of the differences between diseases. Further research addressing the reasons for SPC referral and non-referral, and differences in the preferred and actual place of death in patients with haematological malignancies is also relevant.

\section{CONCLUSIONS}

We have reported evidence of integration between haematology and SPC services, with more referrals among myeloma patients compared to those with AML or DLBCL. We found compelling indications that referral to SPC services was closely associated with death at home, although whether this is a direct result of SPC involvement cannot be determined. Increased integration between clinical haematologists and palliative care specialists may improve end-of-life planning for patients and, particularly, the facilitation of home death when this is the preferred place, even if the disease trajectory mitigates against this for many.

Acknowledgements We are extremely grateful for the legacy that funded this study, and the clinical and administrative teams who facilitate data collection for HMRN. We are indebted to former and current members of the project Steering Group, including: Lee Bond, Caroline Boyd, David Brown, Annette Edwards, Shirley Fraser, Sarah Holmes, Suzanne Kite, Una Macleod, David Mazza and Janet Munro. We are indebted to Clarissa Nolan, Ann Hewison and Paula O'Reilly who collected study data; Will Curson for designing and developing the study database; John Blase who prepared the Figure and provided technical assistance during preparation of the final document; and Georgia Mortzou who helped with data inputting.

Contributors DAH, ER, AGS, MRH and ACG planned the study; DAH managed and implemented the study; AGS and HIW managed and conducted data analysis. MRH, RP, ACG and MJJ provided on-going clinical advice. DAH, AGS and ER wrote the first draft of the paper and are guarantors of the study; all authors contributed to further redrafting of the final manuscript. All authors had full access to all the data in the study and can take responsibility for the integrity of the data and the accuracy of the data analysis.

Funding The Haematological Malignancy Research Network is funded by Leukaemia \& Lymphoma Research (Grant No: 10042). This study received specific funding via a legacy donated to the Haematology Department at York Teaching Hospital NHS Foundation Trust (Grant No: YOR-A0723). The funders did not make any decisions about the study, or have any influence over its management or publication of findings.

\section{Competing interests None.}

Ethics approval The Haematological Malignancy Research Network has ethical approval (REC 04/01205/69) from Leeds West Research Ethics Committee, R\&D approval from each Trust in the Yorkshire and Humber and Yorkshire Coast Cancer Networks, and exemption from Section 251 (formally Section 60) of the Health \& Social Care Act (2001) (PIAG 1-05(h)/ 2007). This specific study also has additional ethical approval (REC 07/H1307/168).

Provenance and peer review Not commissioned; externally peer reviewed.

Open Access This is an Open Access article distributed in accordance with the Creative Commons Attribution Non Commercial (CC BY-NC 3.0) license, which permits others to distribute, remix, adapt, build upon this work noncommercially, and license their derivative works on different terms, provided the original work is properly cited and the use is non-commercial. See: http://creativecommons.org/licenses/by$\mathrm{nc} / 3.0 /$

\section{REFERENCES}

1 Addington-Hall J, Altmann D. Which terminally ill cancer patients in the United Kingdom receive care from community specialist palliative care nurses? J Adv Nurs 2000;32:799-806.

2 Howell DA, Shellens R, Roman E, et al. Haematological malignancy: are patients appropriately referred for specialist palliative and hospice care? A systematic review and meta-analysis of published data. Palliat Med 2011;25:630-41.

3 Auret K, Bulsara C, Joske D. Australasian haematologist referral patterns to palliative care: lack of consensus on when and why. Intern Med J 2003;33:566-71.

4 Cheng W-W, Willey J, Palmer JL, et al. Interval between palliative care referral and death among patients treated at a comprehensive cancer center. J Palliat Med 2005; $8: 1025-32$.

5 Osta BE, Palmer JL, Paraskevopoulos T, et al. Interval between first palliative care consult and death in patients diagnosed with advanced cancer at a comprehensive cancer center. J Palliat Med 2008;11:51-7.

6 Howell DA, Roman E, Cox H, et al. Destined to die in hospital? Systematic review and meta-analysis of place of death in haematological malignancy. BMC Palliat Care 2010;9:9.

7 Cohen J, Bilsen J, Addington-Hall J, et al. Population-based study of dying in hospital in six European countries. Palliat Med 2008;22:702-10.

8 Gomes B, Higginson IJ. Factors influencing death at home in terminally ill patients with cancer: systematic review. $B M J$ 2006;332:515-21.

9 Howell DA, Wang H-I, Smith AG, et al. Place of death in haematological malignancy: variations by disease sub-type and time from diagnosis to death. BMC Palliat Care 2013;12:42.

10 NICE. Improving outcomes in haematological cancers: the manual. London: Department of Health, 2003.

11 Ferlay J, Shin H-R, Bray F, et al. Estimates of worldwide burden of cancer in 2008: GLOBOCAN 2008. Int J Cancer Int Cancer 2010;127:2893-917.

12 Jemal A, Siegel R, Ward E, et al. Cancer statistics, 2008. CA Cancer J Clin 2008;58:71-96.

13 Mechelen WV, Aertgeerts B, Ceulaer KD, et al. Defining the palliative care patient: a systematic review. Palliat Med 2013;27:197-208.

14 Pastrana T, Jünger S, Ostgathe C, et al. A matter of definitionkey elements identified in a discourse analysis of definitions of palliative care. Palliat Med 2008;22:222-32.

15 Costantini M, Ripamonti C, Beccaro M, et al. Prevalence, distress, management, and relief of pain during the last 3 months of cancer patients' life. Results of an Italian mortality follow-back survey. Ann Oncol 2009;20:729-35.

16 Prieto JM, Blanch J, Atala J, et al. Psychiatric morbidity and impact on hospital length of stay among hematologic cancer patients receiving stem-cell transplantation. J Clin Oncol 2002;20:1907-17.

17 Manitta V, Zordan R, Cole-Sinclair M, et al. The symptom burden of patients with hematological malignancy: a cross-sectional observational study. J Pain Symptom Manage 2011;42:432-42.

18 Morselli M, Bandieri E, Zanin R, et al. Pain and emotional distress in leukemia patients at diagnosis. Leuk Res 2010;34: e67-8.

19 Fadul NA, El Osta B, Dalal S, et al. Comparison of symptom burden among patients referred to palliative care with hematologic malignancies versus those with solid tumors. J Palliat Med 2008;11:422-7. 
20 Hung Y-S, Wu J-H, Chang H, et al. Characteristics of patients with hematologic malignancies who received palliative care consultation services in a medical center. Am J Hosp Palliat Care 2013;30:773-80.

21 Niscola P. Effective pain management in hematological malignancies. Expert Rev Hematol 2009;2:219-22.

22 Zimmermann C, Yuen D, Mischitelle A, et al. Symptom burden and supportive care in patients with acute leukemia. Leuk Res 2013;37:731-6.

23 Smith A, Roman E, Howell D, et al. The Haematological Malignancy Research Network (HMRN): a new information strategy for population based epidemiology and health service research. Br J Haematol 2010;148:739-53.

24 Smith A, Howell D, Patmore R, et al. Incidence of haematological malignancy by sub-type: a report from the Haematological Malignancy Research Network. Br J Cancer 2011;105:1684-92.

25 Swerdlow SH, Campo E, Harris NL, et al. WHO classification of tumours of haematopoietic and lymphoid tissues. 4th edn. Lyon, France: International Agency for Research on Cancer, 2008.

26 SAS Institute Inc. SAS statistical analysis software. Cary, NC, USA: SAS Institute Inc., 2011.

27 Tse DMW, Chan KS, Lam WM, et al. The impact of palliative care on cancer deaths in Hong Kong: a retrospective study of 494 cancer deaths. Palliat Med 2007;21:425-33.

28 Hunt RW, Fazekas BS, Luke CG, et al. The coverage of cancer patients by designated palliative services: a population-based study, South Australia, 1999. Palliat Med 2002;16:403-9.

29 Beccaro M, Costantini M, Merlo DF. Inequity in the provision of and access to palliative care for cancer patients. Results from the Italian survey of the dying of cancer (ISDOC). BMC Public Health 2007;7:66.
30 Ansell P, Howell D, Garry A, et al. What determines referral of UK patients with haematological malignancies to palliative care services? An exploratory study using hospital records. Palliat Med 2007;21:487-92.

31 Lidstone V, Butters E, Seed PT, et al. Symptoms and concerns amongst cancer outpatients: identifying the need for specialist palliative care. Palliat Med 2003;17:588-95.

32 Fadul N, Elsayem A, Palmer JL, et al. Predictors of access to palliative care services among patients who died at a comprehensive cancer center. J Palliat Med 2007;10:1146-52.

33 Johnston GM, Gibbons L, Burge FI, et al. Identifying potential need for cancer palliation in Nova Scotia. Can Med Assoc J 1998;158:1691-8.

34 Maddocks I, Bentley L, Sheedy J. Quality of life issues in patients dying from haematological diseases. Ann Acad Med Singapore 1994;23:244-8.

35 Costantini M, Camoirano E, Madeddu L, et al. Palliative home care and place of death among cancer patients: a population-based study. Palliat Med 1993;7:323-31.

36 Grande GE, McKerral A, Todd CJ. Which cancer patients are referred to Hospital at Home for palliative care? Palliat Med 2002;16:115-23.

37 Gomes B, Calanzani N, Curiale V, et al. Effectiveness and cost-effectiveness of home palliative care services for adults with advanced illness and their caregivers. Cochrane Database Syst Rev 2013;(6):CD007760.

38 Becker R. Palliative care. 2: exploring the skills that nurses need to deliver high-quality care. Nurs Times 2009;105: $18-20$.

39 Skilbeck J, Payne S. Emotional support and the role of Clinical Nurse Specialists in palliative care. J Adv Nurs 2003;43:521-30. 